\title{
A classificação industrial e sua importância na análise setorial
}

\author{
Suzana Borschiver \\ Professora adjunta e pesquisadora no Sistema de Informação da \\ Indústria Química/UFRJ - área de gestão e inovação tecnológica. \\ Escola de Química da Universidade Federal do Rio de Janeiro. Centro \\ de Tecnologia. \\ E-mail:suzana@eq.ufrj.br
}

Pedro Wongtschowski

Diretor superintendente da Oxiteno S/A.

E-mail: pedwong@ultra.com.br

\section{Adelaide Antunes}

Professora adjunta e coordenadora no Sistema de Informação da Indústria Química/UFRJ - área de gestão e inovação tecnológica. E-mail: adelaide@eq.ufrj.br

\section{Resumo}

O presente artigo tem como objetivo apresentar conceitos acerca das classificações industriais, mostrando-se a importância dos sistemas de classificações e a necessidade de correlação entre as classificações dos diferentes países. As classificações internacionais são baseadas na "Família Internacional de Classificações Econômicas e Sociais", que englobam atividades econômicas, produtos, consumo, educação, emprego, entre outras. Sua abrangência em relação às classificações de atividades e de produtos envolve as "classificações de referência (como a ISIC e a CPC), as classificações derivadas (como a NACE e a CPA) e as classificações relacionadas (como a ANZIC e a NAICS), cuja harmonização é realizada por gestores de classificação, como o INDEC, da Argentina, o INE, de Portugal, e o IBGE, do Brasil.

As classificações de atividades e de produtos na economia constituem instrumento básico para obtenção de informações e análise dos dados estatísticos.

\section{Palavras-chave}

Classificação industrial; Análise setorial; Informação estatística.

\section{Industrial classification and its importance in analysis by sector}

\begin{abstract}
The objective of the present article is to present concepts related to industrial classification systems and to demonstrate both the importance of classification systems and the necessity of correlation between the classification systems in use in different countries. International classification is based on the "International Family of Economic and Social Classifications", which contains classifications of economic activities, products, consumption, education, employment and social factors, amongst others. The scope of the international family of economic and social classifications in relation to activities and products involves reference classifications, (such as the ISIC and the CPA), derived classifications (such as the NACE and the CPC), and related classifications (such as the ANZIC and the NAICS). The harmonization of these classifications is undertaken by organizations which specialize in classification management, such as INDEC in Argentina, INE in Portugal and IBGE in Brazil.

This being the case, the classifications of economic activities and products constitute a basic tool for obtaining information and analyzing statistical.
\end{abstract}

\section{Keywords}

Industrial classification; Sectorial analysis; Statistical information.

\section{INTRODUÇÃO}

No período compreendido entre os séculos XV e XVIII, conhecido como Idade Moderna, o mundo passou por grandes transformações no âmbito jurídico-político, econômico, social e ideológico, concentradas principalmente na Europa. Na economia, os mercados locais se ampliaram, tornando-se nacionais e, com as grandes navegações, internacionais. As transformações mundiais atravessaram os séculos, e, com elas, a evolução gradativa do comércio foi inevitável. Como conseqüência, em face da diversidade de línguas e costumes, os países procuram, desde aquela época, encontrar uma linguagem comum para identificar os produtos e atividades que fazem parte das suas relações comerciais nacionais e internacionais (Funcex, 1999).

No final do século XX, devido ao grande volume de comércio internacional e à discrepância acentuada entre as estatísticas geradas pelos países, iniciaram-se alguns estudos com o objetivo de unificar as informações comerciais, tanto do ponto de vista estatístico quanto do ponto de vista conceitual. A necessidade de classificar um produto ou uma atividade econômica nasceu da existência do grande número de informações, valores e dados estatísticos gerados pela economia, sejam eles de produção ou serviços, e suas respectivas atividades produtivas, visando ao melhor entendimento das relações comerciais. Em paralelo a um sistema de classificação, a adoção de uma linguagem comum constitui um dos requisitos básicos para construção de um sistema de informação útil para apoiar as análises de diferentes agentes econômicos e facilitar o planejamento de políticas de desenvolvimento econômico que correspondam às reais necessidades dos mercados nacionais e internacionais.

As classificações constituem um sistema lingüístico que se utiliza para comunicar e processar estaticamente os fenômenos. Permitem dividir o universo de dados estatísticos em categorias as mais homogêneas possiveis, pois, nos estudos dos fenômenos econômicos, as informações sobre os acontecimentos devem obedecer a uma ordenação lógica, com coerência nos elementos que estão correlacionados. As nomenclaturas e as notações decimais hierarquizadas dos códigos empregados para definir as atividades e produtos são 
elaboradas visando, principalmente, a facilitar a organização das informações. Uma vez que as classificações apóiam as análises setoriais da economia e tratamento de dados no que concerne à produção, distribuição e comercialização de produtos e serviços, atualmente qualquer tipo de estudo que se faça de setores industriais deve estar apoiado em definições preestabelecidas de quais produtos e/ou atividades neles estão incluídos.

A sistematização de classificação industrial dá-se segundo uma ou mais características dos bens e serviços produzidos: a partir dos usos a que se destinam, e/ou dos insumos utilizados, e/ou da tecnologia empregada e/ou da organização da produção. Desta forma, é possível definir cada atividade da economia, cada produto, tornando comparáveis as informações pertinentes aos produtos e atividades investigadas nos censos e nas demais pesquisas industriais.

Apesar da busca contínua por um sistema de classificações de atividades econômicas e produtos na mesma base conceitual e comparável em todo o mundo, a divergência entre os limites estabelecidos para os setores em que uma economia está dividida dificulta o processo de análise dos dados estatísticos e do comportamento da economia.

Os dados devem ser agrupados segundo categorias cujas características são semelhantes, proporcionando as informações agregadas necessárias para descrever e analisar a estrutura, funcionamento e inter-relações setoriais da economia como um todo (IBGE, 1988).

Retratar adequadamente os diversos fenômenos de um mundo cada vez mais complexo não é tarefa trivial, exigindo, antes de tudo, uma abordagem multidisciplinar. A era do conhecimento está transformando a indústria. Neste novo modelo, a informação assume papel de destaque, uma vez que é base para o conhecimento. O domínio na produção, o tratamento, a armazenagem e a disseminação de informações (conhecimento), portanto, podem ser consideradas vantagens competitivas para os tomadores de decisão dos diferentes países (Valente, 1996).

\section{A INFORMAÇÃO ESTATÍSTICA}

Nas sociedades modernas, a informação constitui, primordialmente, o elemento básico para a tomada de decisões. No entanto, os dados que representam os fatos econômicos e sociais só têm significância, ou seja, só transformam-se em informação, se ordenados, sistematizados, incluídos em esquemas de classificação (Valente, 1996).
O desenvolvimento e aplicação de modelos de classificação nos diversos âmbitos da indústria, nos sistemas tecnológicos e até nas políticas científicas e tecnológicas constituem elementos de grande importância dentro das tendências recentes de estudos setoriais (Porcaro, 2000). Todos os processos econômicos que devem ser descritos mediantes estatísticas requerem uma classificação sistemática. A classificação oficial de um país faz parte do chamado Sistema de Informação Estatística (SIE). Este sistema pode ser visto como uma organização específica da sociedade e compreende o conjunto de informações estatísticas oficiais coletadas, tratadas, organizadas, analisadas e disseminadas por um órgão estatístico central, visando ao conhecimento da realidade econômica e social do país (Desrosières, 1992).

Portanto, as classificações são instrumentos de base que determinam substancialmente a qualidade das informações estatísticas, sem os quais estas informações não poderiam ser elaboradas. A elaboração e revisão periódica das nomenclaturas estatísticas e econômicas constituem um desafio permanente devido à evolução constante da estrutura econômica que gera novas atividades econômicas e novos produtos e reduz a importância de algumas atividades e bens existentes. Os intervalos entre as revisões não podem ser demasiadamente longos, visto que, com o tempo, as classificações se tornam menos adequadas, mas também não podem ser demasiadamente curtos, senão prejudicarse-ia a comparabilidade dos dados ao longo do tempo*. Toda a revisão de uma classificação, especialmente quando implica alterações de tipo estrutural, conduz forçosamente a rupturas nas séries temporais. A tabela 1, a seguir, exemplifica a periodicidade de revisão de algumas das classificações utilizadas nas classificações internacionais de atividades e produtos.

Para elaborar estatísticas comparáveis aos níveis internacionais, exigem-se, naturalmente, não só a aplicação de definições e conceitos estatísticos homogêneos, mas também a harmonização das nomenclaturas utilizadas. Por isso, uma das tarefas prioritárias dos serviços internacionais de estatística é a elaboração de nomenclaturas adequadas ou a revisão das existentes. No caso brasileiro, o órgão estatístico central é o Instituto Brasileiro de Geografia e Estatística (IBGE),

\footnotetext{
* Algumas classificações possuem periodicidade nos intervalos de suas revisões e dispõem do status da revisão pela qual estejam passando. Estas observações encontram-se no site da United Nations Statistics Division disponível: http:// www.un.org/ (acessado em 04/ 07/2001).
} 
criado por legislação oficial específica e definido como órgão coordenador e principal produtor do Sistema Estatístico Nacional (SEN). Integram o SEN os órgãos e entidades da administração pública direta e indireta, dos âmbitos federais, estaduais e municipais, produtores de estatísticas. No próximo item, serão abordadas as classificações existentes no mundo e como os trabalhos de normalização das classificações têm sido orientados pelos órgãos responsáveis por estes trabalhos, os gestores de classificação.

\section{FAMÍLIA INTERNACIONAL DE CLASSIFICAÇÕES ECONÔMICAS}

No mundo, os países utilizam como padrão de referência para classificações a Família Internacional de Classificações Econômicas e Sociais* (List of International Family of Economic and Social Classification ${ }^{* *}$ ). Nela estão contidas classificações de atividades econômicas, de produtos, de consumo, de educação, de emprego, sociais, entre outras.

As classificações de atividades econômicas são construídas para organizar as informações das unidades produtivas e institucionais com o objetivo de produzir estatísticas dos fenômenos derivados da sua participação no processo econômico, a partir de ordenamento que privilegia a identificação de segmentos homogêneos quanto à tecnologia, mercado e organização de produção. Assim, constituem instrumento básico para assegurar a coordenação entre a coleta, a apresentação e a análise dos dados estatísticos. Por outro lado, a articulação das estatísticas no tempo (séries temporais), no espaço (nas comparações internacionais e subnacionais) e entre fontes diversas (integração dos sistemas nacionais) tem como premissa o uso de classificações uniformes, ou seja, a adoção de uma linguagem comum (Schwartzman, 1997).

De maneira geral, as atividades econômicas podem ser definidas como conjuntos de agentes do processo de produção; e os produtos, como conjuntos de bens e serviços.

\footnotetext{
* United Nations, 1996. Relatório do Grupo de Especialistas em Classificações Internacionais Econômicas e Sociais. International Economic and Social Classifications(E/CN.3/1997/4).

** United Nations, 1998. Este texto foi revisto de forma preliminar no Quarto Encontro do Grupo de Especialistas em Classificações Internacionais Econômicas e Sociais, Nova York, 2 a 4 de novembro de 1998 (preparado pela Divisão de Estatística das Nações Unidas). Expert Group Meeting on Applying New Methods and Technologies (ESA/ STAT/AC.63/18).
}

TABELA 1

Período de revisão das classificações internacionais de produtos e atividades econômicas

\begin{tabular}{|c|c|}
\hline Classificação & Período de Revisão \\
\hline $\begin{array}{l}\text { International Standard Industrial } \\
\text { Classification of All Economic Activities (Isic) }\end{array}$ & Irregular \\
\hline $\begin{array}{l}\text { Nomenclature Générale des Activités } \\
\text { Économiques dans les Communautées } \\
\text { Européennes (Nace) }\end{array}$ & Irregular \\
\hline $\begin{array}{l}\text { North American Industry Classification } \\
\text { System (NAICS) }\end{array}$ & $5-10$ anos \\
\hline $\begin{array}{l}\text { Australian and New Zealand Industrial } \\
\text { Classification (ANZSIC) }\end{array}$ & 10 anos \\
\hline Central Product Classification (CPC), & 8 anos \\
\hline $\begin{array}{l}\text { Harmonized Commodity Description and } \\
\text { Coding System (HS) }\end{array}$ & $4-6$ anos \\
\hline $\begin{array}{l}\text { Standard International } \\
\text { Classification (SITC) }\end{array}$ & 10 anos \\
\hline $\begin{array}{l}\text { Classification by Broad Economic } \\
\text { Categories Defined in terms of Standard } \\
\text { International Trade Classification (BEC) }\end{array}$ & Irregular \\
\hline Classification of Products by Activity (CPA) & 5 anos \\
\hline
\end{tabular}

Fonte: elaboração própria a partir do site da United Nations disponível:http://www.un.org/ (acesso em 04/07/2001).

As atividades industriais podem ser de diferentes modalidades: produtivas, ou seja, de bens e serviços de manutenção/reparação de máquinas e equipamentos e de apoio; atividades diretamente associadas ao processo produtivo, como produção de utilidades, prestação de serviços de controle de qualidade, desenvolvimento de projetos e tratamento de poluentes, e atividades indiretamente associadas à produção, como as administrativas e auxiliares (IBGE, 1999). Por suas vez, as classificações de produtos servem como instrumento para o agrupamento destes segundo as características físicas, tipos de insumo e etapas do processo produtivo (classificações de comércio exterior), origem no processo produtivo (classificações produto/atividade) e mercado ou finalidade de uso (classificações de consumo por função), bem como ponto de partida básico para a preparação de estatísticas e análise dos fluxos de bens e serviços na economia. Tais estatísticas podem cobrir a produção, consumo intermediário* e final, formação de capital, comércio exterior ou preços. Também podem

\footnotetext{
* Significa consumo industrial, ou seja, o que é consumido pelas indústrias para gerar o produto final.
} 
estar referidas a fluxos de produtos, balanços e podem ser compiladas no contexto de produção de tabelas de insumo-produto e da balança de pagamentos.

\section{Abrangência da Família Internacional de Classificações}

A Família Internacional de Classificações abrange as classificações registradas no United Nations Inventory of Classification, revisadas e aprovadas como diretrizes pela United Nation Statistical Commission ou outro órgão intergovernamental competente em questões como economia, trabalho demográfico, saúde, educação, bemestar social, geografia, meio ambiente e turismo*. Também inclui as classificações derivadas ou relacionadas às classificações internacionais e principalmente, mas não unicamente, usadas para propósitos regionais ou nacionais. A Família engloba três tipos principais de classificações: de referência, derivada e relacionada (United Nations, 1999).

1. As classificações de referência são resultantes dos acordos internacionais aprovados pela United Nation Statistical Commission ou outro órgão intergovernamental competente, tais como o International Labour Organization (ILO), o International Monetary Fund (IMF), o United Nations Educational, Scientific and Cultural Organization (Unesco), o World Health Organization (WHO) ou a World Customs Organization (WCO), dependendo do tema da área. Dessa maneira, as classificações de referência alcançaram uma ampla aceitação e concordância oficial e são aprovadas e recomendadas como referências (guidelines) para o preparo de classificações. Elas podem ser usadas como modelos para o desenvolvimento ou revisão de outras classificações, tanto em relação à estrutura quanto em relação ao caráter e definição das categorias.

2. As classificações derivadas são baseadas nas classificações de referência. As classificações derivadas podem ser preparadas tanto pela adoção das categorias e da estrutura das classificações de referência, às vezes incluindo um detalhamento adicional além do fornecido pela classificação de referência, ou podem ser preparadas por meio da reorganização ou agregação de itens de uma ou mais classificações de referência. Um exemplo de classificação derivada é a Nomenclature Générale des Activités Économiques dans les Communautées Européennes (Nace), que é baseada na International Standard Industrial Classification of All Economic Activities (ISIC).

* A Família Internacional de Classificações engloba também as classificações sociais que não fazem parte do contexto do estudo deste trabalho.
3. As classificações relacionadas referem-se parcialmente às classificações de referência, ou são associadas a estas apenas em alguns níveis específicos de sua estrutura*.

Os procedimentos de manutenção, atualização e revisão das classificações estatísticas da Família Internacional incentivam a resolução de problemas de correspondência parcial entre as classificações relacionadas e fornecem a oportunidade para se aumentar a harmonização.

As classificações estatísticas internacionais de referência e suas respectivas classificações derivadas e relacionadas são mutuamente dependentes. A existência de um sistema de classificação internacional de referência que reflete com clareza o entendimento da sua área de aplicação facilita o trabalho de desenvolvimento e revisão dos sistemas de classificações derivados e relacionados. A colaboração entre as agências/gestores de classificação é necessária para harmonização das mesmas.

\section{Gestores de Classificação para Cooperação Internacional}

As classificações estatísticas das Nações Unidas são as utilizadas atualmente pela maioria dos países. Uma parte do trabalho de cooperação internacional em classificações é assegurar harmonização e padronização, de modo que as classificações estatísticas no nível internacional sejam comparáveis dos níveis regionais ao nível nacional. Isto é realizado por meio da troca de informações em reuniões, escritórios regionais, intercâmbio entre os países, assim como por interação entre os órgãos estatísticos internacionais.

$\mathrm{Na}$ tabela 2, a seguir, são listados os principais órgãos estatísticos nacionais e internacionais.

As instituições com a responsabilidade da manutenção, atualização e revisão das classificações são denominadas órgãos estatísticos centrais ou gestores de classificações. Cada gestor fica com a responsabilidade de conduzir a manutenção, atualização e revisão de sua(s) própria(s) classificação(ões). Ao mesmo tempo, o gestor pode ter o conhecimento sobre, ou participar de certos aspectos das atividades de manutenção, atualização e revisão de outras classificações da família. A tabela 3, a seguir, mostra o exemplo de gestores de algumas classificações.

* São inúmeros exemplos de correlação das classificações que podem ser visualizadas no site da United Nations, disponível em: http://www.un.org/ (acessado em 04/07/2001). 
TABELA 2

\section{Principais Órgãos Estatísticos Nacionais e Internacionais}

\begin{tabular}{|c|c|}
\hline \multicolumn{2}{|l|}{ AMÉRICA } \\
\hline ARGENTINA & Instituto Nacional de Estadística y Censos (Indec) \\
\hline BOLÍVIA & Instituto Nacional de Estadística (INE) \\
\hline BRASIL & $\begin{array}{l}\text { Instituto Brasileiro de Geografia e Estatística } \\
\text { (IBGE) }\end{array}$ \\
\hline COLÔMBIA & $\begin{array}{l}\text { Departamento Administrativo Nacional de } \\
\text { Estadistica (DANE) }\end{array}$ \\
\hline CHILE & Instituto Nacional de Estadísticas (INE) \\
\hline EQUADOR & Instituto Nacional de Estadística y Censos (Indec) \\
\hline PARAGUAI & $\begin{array}{l}\text { Direccion General de Estadistica, Encuestas y } \\
\text { Censos (DGEEC) }\end{array}$ \\
\hline PERU & Instituto Nacional de Estadística e Informática (Inei) \\
\hline URUGUAI & Instituto Nacional de Estadística (INE) \\
\hline VENEZUELA & Oficina Central de Estadística e Informatica (Ocei) \\
\hline MÉXICO & $\begin{array}{l}\text { Instituto Nacional de Estadística, Geografia e } \\
\text { Informatica (Inegi) }\end{array}$ \\
\hline GUATEMALA & Instituto Nacional de Estadística (INE) \\
\hline COSTA RICA & Instituto Nacional de Estadística y Censos (Inec) \\
\hline EUA & U. S. Bureau of the Census \\
\hline CANADÁ & Statistics Canada \\
\hline \multicolumn{2}{|l|}{ EUROPA } \\
\hline PORTUGAL & Instituto Nacional de Estadística (INE) \\
\hline ESPANHA & Instituto Nacional de Estadística (INE) \\
\hline FRANÇA & Institut National d'Études Démographiques (Ined) \\
\hline FRANÇA & $\begin{array}{l}\text { Institut Nacional de la Statistique e d'Études } \\
\text { Economiques (Insee) }\end{array}$ \\
\hline ITÁlIA & Instituto Nazionale di Statistica (Istat) \\
\hline FINLÂNDIA & Instituto Nazionale di Statistica (Istat) \\
\hline HOLANDA & Statistics Netherlands \\
\hline DINAMARCA & Statistik Denmarks \\
\hline NORUEGA & Statistics Norway \\
\hline SUÍÇA & Statistics Sweden \\
\hline \multicolumn{2}{|c|}{ ÁSIA / ÁFRICA / OCEANIA } \\
\hline CHINA & State Statistical Bur \\
\hline JAPÃO & Statistics Bureau and Statistics Center \\
\hline INDONÉSIA & Central Bureau of Statistics \\
\hline ÍNDIA & Census of India \\
\hline CORÉIA & National Statistical Office \\
\hline ISRAEL & Central Bureau Statistics (CBS) \\
\hline MOÇAMBIQUE & National Institute of Statistics \\
\hline CABO VERDE & Instituto Nacional de Estatística \\
\hline ZÂMBIA & The Central Statistical Office Republic of Zambia \\
\hline AUSTRÁLIA & Australian Bureau of Statistics (ABS) \\
\hline NOVA ZELÂNDIA & Statistics New Zealand \\
\hline \multicolumn{2}{|l|}{ ORGANIZAC̣ÕES } \\
\hline ONU & United Nations \\
\hline EUROSTAT & Statistical Office of the European Communities \\
\hline WORLD BANK & Banco Mundial \\
\hline CEPAL & Comision Economica para America Latina y el Cari \\
\hline CELADE & Comision Latinoamericano de Demografia \\
\hline
\end{tabular}

Fonte: elaboração própria a partir de dados do IBGE, Classificações Estatísticas 2001.

\section{TABELA 3}

Gestores das principais classificações da Família Internacional de Classificações

\begin{tabular}{|c|c|}
\hline CLASSIFICAC̣̃̃O & GESTOR \\
\hline $\begin{array}{l}\text { Isic (International Standard Industrial } \\
\text { Classification of All Economic Activities) }\end{array}$ & $\begin{array}{l}\text { United Nations } \\
\text { Statistics Division }\end{array}$ \\
\hline $\begin{array}{l}\text { Nace (Nomenclature Générale des } \\
\text { Activités Économiques dans les } \\
\text { Communautées Européennes) }\end{array}$ & $\begin{array}{l}\text { Statistical Office of the } \\
\text { European } \\
\text { Communities }\end{array}$ \\
\hline $\begin{array}{l}\text { Naics - Canada (North American Industry } \\
\text { Classification System) }\end{array}$ & Statistics Canada \\
\hline $\begin{array}{l}\text { Naics - México (North American Industry } \\
\text { Classification System) }\end{array}$ & $\begin{array}{l}\text { Instituto Nacional de } \\
\text { Estadística, Geografia e } \\
\text { Informatica }\end{array}$ \\
\hline $\begin{array}{l}\text { Naics - USA (North American Industry } \\
\text { Classification System) }\end{array}$ & $\begin{array}{l}\text { US Bureau of the } \\
\text { Census }\end{array}$ \\
\hline $\begin{array}{l}\text { ANZSIC (Australian and New Zealand } \\
\text { Standard Industrial Classification) }\end{array}$ & $\begin{array}{l}\text { Statistics New Zealand } \\
\text { / Australian Bureau of } \\
\text { Statistics }\end{array}$ \\
\hline CPC (Central Product Classification) & $\begin{array}{l}\text { United Nations } \\
\text { Division Statistics }\end{array}$ \\
\hline $\begin{array}{l}\text { HS (Harmonized Commodity Description } \\
\text { and Coding System) }\end{array}$ & $\begin{array}{l}\text { World Customs } \\
\text { Organization }\end{array}$ \\
\hline $\begin{array}{l}\text { SITC Rev } 3 \text { (Standard International Trade } \\
\text { Classification) }\end{array}$ & $\begin{array}{l}\text { United Nations } \\
\text { Division Statistics }\end{array}$ \\
\hline $\begin{array}{l}\text { BEC Rev } 3 \text { (Classification by Broad } \\
\text { Economic Categories in Terms of the } \\
\text { Standard International Trade Classification) }\end{array}$ & $\begin{array}{l}\text { United Nations } \\
\text { Division Statistics }\end{array}$ \\
\hline $\begin{array}{l}\text { CPA (Classification of Products by } \\
\text { Activity) }\end{array}$ & $\begin{array}{l}\text { Statistical Office of the } \\
\text { European Communities }\end{array}$ \\
\hline
\end{tabular}

Fonte: elaboração própria a partir de dados do site da United Nations, disponível em: http://www.un.org/ (acesso em 04/07/2001).

Para a harmonização das classificações, é necessária a colaboração entre os gestores. Os gestores internacionais podem ser definidos como Gestores das Classificações de Referência, que são as agências internacionais responsáveis pela manutenção, atualização e revisão das classificações de referência ou classificações estatísticas internacionais, Gestores das Classificações Estatísticas Derivadas, que são agências ou órgãos estatísticos que fazem uso das classificações de referência no preparo e manutenção de classificações derivadas, e Gestores das Classificações Estatísticas Relacionadas, que são agências ou órgãos estatísticos que preparam e fazem a manutenção de classificações que são referidas a uma classificação de referência. 


\section{A harmonização das classificações}

O objetivo da harmonização é tornar os dados estatísticos comparáveis, mesmo que sejam gerados por diferentes localizações geográficas e/ou diferentes métodos e unidades estatísticas. Isto é possível por meio do uso de classificações harmonizadas ou de sistemas de classificações comparáveis.

A harmonização de classificações estatísticas requer um processo de conciliação das diferentes classificações em uma estrutura comum, buscando aumentar ao máximo a correspondência entre elas. Isto inclui o uso da mesma base conceitual e terminologia, assim como o estabelecimento de tabelas de correspondência entre as categorias das diferentes classificações, ou a identificação detalhada da construção destas categorias.

O trabalho de harmonização requer uma troca regular de informações entre os órgãos estatísticos juntamente com a coordenação e co-participação de comitês e grupos de tarefas de modo a estabelecer sistemas de classificações de referência, derivados e relacionados na mesma base conceitual. Os sistemas de classificações derivados, por exemplo, possuem a mesma estrutura do sistema de classificações de referência internacionais, ainda que sejam definidas categorias mais detalhadas além da estrutura já existente, ao passo que as classificações relacionadas divergem das classificações de referência sob algum aspecto, como, por exemplo, seguindo sua estrutura em apenas um nível. A extensão da possível harmonização e correspondência entre as diferentes classificações depende do grau de semelhança entre as categorias e das suas bases conceituais. A necessidade de comparabilidade entre as informações estatísticas produzidas por dois países pode ser atendida pela simples correlação entre seus respectivos sistemas de classificações nacionais. Entretanto, um sistema de classificação comum é necessário para a análise das relações econômicas entre mais de dois países simultaneamente.

Quando as classificações internacionais diferem da classificação nacional, a comparação internacional pode ser realizada pelo rearranjo das categorias do sistema nacional de acordo com o internacional, lembrando que é necessário estabelecer um elo entre as estatísticas nacionais e o sistema internacional correspondente.

A tabela 4 ilustra os principais tipos de classificações da Família Internacional de Classificações.
TABELA 4

Principais classificações da Família Internacional de Classificações

\begin{tabular}{|c|c|c|}
\hline & Atividade Econômica & Produto \\
\hline Referência & $\begin{array}{l}\text { International Standard } \\
\text { Industrial Classification of } \\
\text { All Economic Activities } \\
\text { Revision } 3 \text { (ISIC Rev 3) } \\
\text { ISIC Rev } 2 \\
\text { ISIC Rev } 1 \\
\text { ISIC Original Version } 1948\end{array}$ & $\begin{array}{l}\text { Central Product } \\
\text { Classification (CPC) } \\
\text { Version } 1.0 \\
\text { Harmonized } \\
\text { Commodity } \\
\text { Description and } \\
\text { Coding System (HS) }\end{array}$ \\
\hline Derivada & $\begin{array}{l}\text { Nomenclature Générale } \\
\text { des Activités Économiques } \\
\text { dans les Communautées } \\
\text { Européennes (NACE Rev 1) }\end{array}$ & $\begin{array}{l}\text { Standard International } \\
\text { Trade Classification } \\
\text { Revision } 3 \text { (SITC Rev 3) } \\
\text { Classification by Broad } \\
\text { Economic Categories } \\
\text { Defined in terms of SITC } \\
\text { Revision } 3 \text { (BEC Rev 3) } \\
\text { Classification of Products } \\
\text { by Activity (CPA) }\end{array}$ \\
\hline Relacionada & $\begin{array}{l}\text { Australian and New Zealand } \\
\text { Industrial Classification } \\
\text { (ANZSIC) } \\
\text { North American Industry } \\
\text { Classification System } \\
\text { (NAICS) } \\
\text { NAICS - Canadá } \\
\text { NAICS - México } \\
\text { NAICS - USA }\end{array}$ & \\
\hline
\end{tabular}

Fonte: elaboração própria a partir de dados do site da United Nations disponivel em: http://www.un.org/ (acesso em 04/07/2001).

\section{Principais Classificações Internacionais de} Atividades Econômicas e de Produtos

\section{Classificações de Atividades Econômicas}

- International Standard Industrial Classification of All Economic Activities (ISIC)

A International Standard Industrial Classification of All Economic Activities (ISIC) (United Nations, 1999) é uma classificação de atividades econômicas de referência que tem como gestor a United Nations Statistics Division (UNSD) utilizada para classificar dados estatísticos nos campos da população, produção, emprego e outras estatísticas econômicas. A ISIC tem por finalidade principal estabelecer uma classificação uniforme das atividades econômicas produtivas. A ISIC já passou por três revisões desde 1948, data de sua implantação. As revisões periódicas da ISIC ocorreram devido às mudanças na economia com o surgimento de novas atividades econômicas e extinção de outras, levando-se em consideração a manutenção da comparabilidade entre as revisões precedentes. 
Atualmente, a estrutura do sistema de classificação da ISIC encontra-se dividida de acordo com a tabela 5. Os principais critérios empregados para definir as divisões e grupos (as categorias de 2 e 3 dígitos respectivamente) da ISIC se referem às características das atividades das unidades produtivas que têm um caráter estratégico para determinar o grau de analogia da estrutura das unidades produtivas e certas relações dentro da economia.

A estrutura e a definição das atividades da ISIC servem como modelo na elaboração de outras classificações. Dessa classificação, por exemplo, foi elaborada a Classificação Européia, Nomenclature Générale des Activités Économiques dans les Communautées Européennes (NACE), o International Labour Organization (ILO), a Food and Agriculture Organization $(\mathrm{FAO})$ e a United Nations Educational Scientific and Cultural Organization (Unesco).

A seguir, exemplifica-se a estrutura do sistema de classificação ISIC através dos níveis compreendidos na categoria Indústria de Transformação (quadro 1).

- Nomenclature Générale des Activités Économiques dans les Communautées Européennes (NACE)

Em 1970, foi elaborada a Nomenclature Générale des Activités Économiques dans les Communautées Européennes (NACE), que constitui, tal como o nome indica, uma nomenclatura de toda a gama de atividades econômicas.

Até então, a maior parte dos países da Europa trabalhava com nomenclaturas de atividades que haviam sido concebidas de acordo com critérios nacionais específicos, o que aumentava as dificuldades de produção de dados sobre as atividades que fossem comparáveis entre os diversos países.

Conseqüentemente, verificou-se a necessidade de dados sobre as atividades econômicas comparáveis entre todos os estados-membros da União Européia, constatando-se que tais dados só poderiam ser produzidos se existisse uma nomenclatura harmonizada. A partir daí foi elaborada uma nomenclatura comunitária das atividades econômicas que pudesse servir de base para a coleta e apresentação de estatísticas relacionadas com as atividades.

Em 1990, um grupo de trabalho formado pelo Statistical Office of the European Communities (Eurostat, 2001) e por representantes dos estados-membros elaborou a NACE Rev 1, que substituiu a NACE 70, com base na estrutura da ISIC Rev 3, constituindo uma versão mais
TABELA 5

Estrutura do sistema de classificação ISIC

\begin{tabular}{llc}
\hline Nivel & Codificação & No de níveis \\
\hline Categoria & 1 caractere alfabético $(\mathrm{A}-\mathrm{Q})$ & 17 \\
Divisão & 2 dígitos & 60 \\
Grupo & 3 dígitos & 159 \\
Classe & 4 dígitos & 292 \\
\hline
\end{tabular}

Fonte: elaboração própria a partir de dados do United Nations disponivel em: http://www.un.org/ (acesso em 04/07/2001).

\section{QUADRO 1 \\ Exemplificação da estrutura da ISIC}

D - Indústrias de Transformação (CATEGORIA)

24 - Manufaturas de produtos químicos (DIVISÃO) 241 - Produção de químicos básicos (GRUPO) 2411 - Produção de químicos básicos exceto fertilizantes e compostos nitrogenados (CLASSE)

Fonte: United Nations. United Nations Statistics Division, 2001.

detalhada adaptada à especificidade européia. $O$ novo sistema contempla o contexto atual das atividades econômicas e impõe o uso uniforme da nova nomenclatura em todos os estados-membros. Esta nova classificação define a estrutura de todas as nomenclaturas de atividades na União Européia e tem sido adotada por um grande número de outros estados europeus, entre eles alguns países da Europa Oriental e Central, estabelecendo um sistema estatístico comum em toda a Europa.

A NACE Rev 1 é uma classificação derivada da ISIC Rev 3, pois apresenta a mesma estrutura nos níveis "seção"e "divisão", porém possui detalhes adicionais e tem como gestor o Statistical Office of the European Communities.

Uma vez que a NACE Rev 1 reflete as estruturas nacionais, ela inclui não só as atividades importantes comuns a todos os estados-membros, mas também as atividades cuja importância difere de país para país.

Um fato interessante diz respeito à influência das entidades de classes* em relação à estrutura do sistema de classificação, que, no caso da NACE Rev 1, contou com a participação destas dando origem a mais um nível de detalhamento na sua estrutura, que diferentemente da ISIC apresenta o nível "subseção".

\footnotetext{
* No Brasil, pode-se citar como exemplos a Associação Brasileira da Indústria Química (Abiquim) e a Associação Brasileira da Indústria de Química Fina (Abifina), que participaram ativamente da definição da CNAE brasileira.
} 
Assim sendo, a NACE Rev 1 é composta de seção, divisão, grupo e classe, podendo apresentar subclasses conforme as peculiaridades das indústrias de cada país.

A seguir, é exemplificada a estrutura do sistema de classificação Nomenclature d'Activités Française (NAF) utilizado na indústria química francesa (INSEE, 2001) (quadro 2).

\section{- North American Industry Classification System (NAICS)}

O mais atual sistema de classificação industrial norteamericano (United Nations, 1999), o North American Industry Classification System (NAICS), foi desenvolvido em conjunto pelo U.S. Economic Classification Policy Committee (ECPC) do Office of Management and Budget (OMB), Statistics Canada e Instituto Nacional de Estadística, Geografia e Informática do México (Inegi), utilizando a ISIC Rev 3 como referência, em substituição ao sistema existente em cada um destes países, respectivamente, o Standard Industrial Classification (SIC), do Canadá (1980), o Mexican Classification of Activities and Products, do México (1994) e o Standard Industrial Classification (SIC), dos Estados Unidos (1987). O objetivo principal foi proporcionar uma base conceitual comum para coleta, análise e disseminação de estatística industrial utilizada pelo governo, pela universidade, pela comunidade do negócio e pelo público em geral nos três países.

O atual sistema entrou em vigor, no Canadá e nos EUA, no ano de 1997, e, no México, no ano de 1998 (Statistics Canada, 2001).

A elaboração do NAICS levou em conta também as necessidades estatísticas do Acordo de Livre Comércio das Américas (Alca), assinado em janeiro de 1994, que previa o desenvolvimento da classificação industrial comum ao Canadá, ao México e aos EUA* (Statistics Canada, 2001).

Por exemplo, um estudo (Inegi, 1996/1997) de conciliação estatística do comércio México-EUA - cujas relações comerciais vêm aumentando com destaque desde 1990 - realizado em 1996-1997 pelo Instituto Nacional de Estadística, Geografia e Informática do México (Inegi), órgão estatístico mexicano, mostrou que a divergência entre as informações do fluxo comercial destes dois países foi devida, principalmente, às diferentes coberturas geográficas, classificações e metodologias de registro das informações.

* Os EUA, o Canadá e o México compõem o bloco econômico denominado North American Free Trade Agreement (Nafta).

\section{QUADRO 2}

\section{Exemplificação da estrutura da NAF}

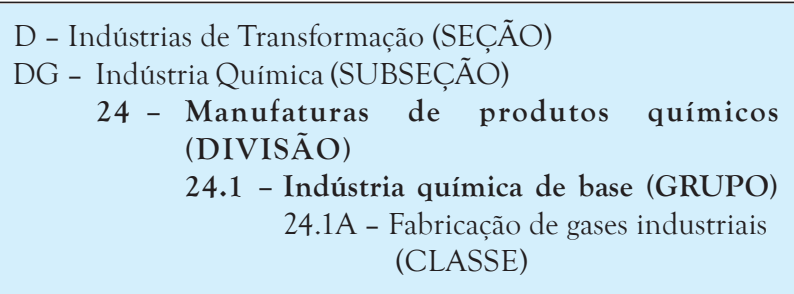

Fonte: INSEE - Institut Nacional de la Statistique e d'Études Economiques. NAF, 2001.

As estatísticas compiladas pelo sistema NAICS são comparáveis com as estatísticas compiladas de acordo com a International Standard Industrial Classification of United Nations (ISIC Rev 3). Desta forma, as classificações industriais podem ser comparadas não somente entre os EUA, o Canadá e o México, mas também com outros países que utilizem a ISIC Rev 3 (Statistics Canada, 2001).

Além disso, o sistema NAICS fornece uma estrutura conceitual baseada no agrupamento de unidades produtivas de acordo com a semelhança dos processos de produção de seus produtos e serviços. A utilização das unidades produtivas como base para a produção dos dados estatísticos permite trazer informações tanto da produção quanto do consumo, tornando a compilação de dados de produção mais adequados.

Um sistema de classificação orientado pela produção ou baseado no consumo, tal como o NAICS, assegura que as agências estatísticas gerem informações que reflitam o desenvolvimento da tecnologia e a diversificação dos serviços existentes no país. Os exemplos mais comuns do uso dos dados estatísticos incluem a medição de produtividade, custos de mão-deobra e construção de tabelas de recursos e usos.

O NAICS-Mexico, o NAICS-Canada e o NAICS-USA são classificações relacionadas e têm como gestores, respectivamente, o Instituto Nacional de Estadística, Geografia e Informática do México (Inegi), o Statistics Canada e os US Bureau of the Census. Como ocorre em outras classificações, a estrutura do sistema NAICS é dividida em níveis. Este sistema de classificação está dividido em 20 setores que podem ser desagregados de acordo com a característica do setor industrial de cada país, obedecendo aos acordos estabelecidos entre os gestores.

Os dados estatísticos podem ser comparados entre os EUA, o Canadá e o México até o nível de "Indústria NAICS". No nível "Indústria Nacional NAICS", as 
classificações variam de acordo com o tipo de organização industrial de cada país. A comparabilidade entre o sistema NAICS do Canadá e dos EUA é maior devido à semelhança existente entre as suas organizações. A tabela 6 apresenta um comparativo da estrutura do sistema NAICS para o Canadá e os EUA.

Alguns símbolos podem indicar comparabilidade entre os EUA, o Canadá e o México. Por exemplo, um retângulo na frente de uma classificação indica comparabilidade entre os três países, ou seja, esta classificação pode ser utilizada pelos órgãos estatísticos dos três países para compilação de dados estatísticos comparáveis. Além disso, quando uma classificação no Canadá é exatamente a mesma indicada nos EUA, utiliza-se como símbolo um diamante na frente da classificação canadense para indicar esta relação (Bureau of Census, 2001).

Em geral, o uso do mesmo código indica que o setor, subsetor, grupo de indústria, indústria ou indústria nacional são comparáveis, mesmo que o título usado nos três países possa diferir em virtude das diferenças no uso da linguagem. O quadro 3 exemplifica a estrutura do NAICS-USA.

- Australian and New Zealand Industrial Classification (ANZSIC)

A Australian and New Zealand Industrial Classification (ANZSIC) foi elaborada em 1992 pelo Statistics New Zealand e pelo Australian Bureau of Statistic para a coleta, compilação e publicação de estatística industrial (Statistics New Zealand, 2001).

Em 1996, uma nova revisão da ANZSIC foi desenvolvida como parte de um esforço cooperativo do Statistics New Zealand com o Australian Bureau of Statistics com o objetivo de harmonizar toda a estatística dos dois países relacionada à indústria. A ANZSIC 96, baseada na ISIC Rev 3, substitui a antiga ANZSIC 93, proporcionando maior cobertura e detalhamento das atividades industriais, assim como comparabilidade dos dados estatísticos gerados na Nova Zelândia, na Austrália e em outros países que igualmente utilizam o sistema ISIC Rev 3 como referência para classificação. A ANZSIC 96 tem como gestores o Statistics New Zealand com o Australian Bureau of Statistic.

A classificação ANZSIC 96 apresenta uma estrutura que abrange quatro níveis: divisão, subdivisão, grupo e classe (tabela 7).

Ci. Inf., Brasília, v. 33, n. 1, p. 9-21, jan./abril 2004

TABELA 6

Comparativo da estrutura hierárquica do NAICS Canadá e EUA

\begin{tabular}{llcc}
\hline Nivel & Código & NAICS - Canadá & NAICS - EUA \\
\hline Setor & 2 dígitos & 20 & 20 \\
Subsetor & 3 dígitos & 99 & 96 \\
Grupo de Indústria & 4 dígitos & 321 & 311 \\
Indústria NAICS & 5 dígitos & 734 & 721 \\
Indústria Nacional & 6 dígitos & 921 & 1.170 \\
\hline
\end{tabular}

Fonte: Statistics Canada, 2001.

\section{QUADRO 3}

Exemplificação da estrutura da NAICS adotada nos EUA
31-33 - Indústria de Transformação (SETOR*)
325 - Indústria Química (SUBSETOR*)
3251 - Indústria química de base (GRUPO DE INDÚSTRIA*)
32513 - Indústria de pigmentos e corantes sintéticos (INDÚSTRIA*)
325131 - Indústria de pigmentos e corantes inorgânicos (INDÚSTRIA NACIONAL)
* Categorias comparáveis com NAICS-Canada e NAICS-Mexico. A classificação no nível INDÚSTRIA NACIONAL varia de país para país, conforme a organização industrial de cada um.

Fonte: Bureau of Census, USA, 2001.

\section{TABELA 7}

Estrutura do sistema de classificação ANZSIC

\begin{tabular}{llc}
\hline Nivel & Codificação & No de niveis \\
\hline Divisão & 1 caractere alfabético (A-Q) & 17 \\
Subdivisão & 2 dígitos & 53 \\
Grupos & 3 digitos & 158 \\
Classe & 4 digitos & 465 \\
\hline
\end{tabular}

Fonte: elaboração própria a partir de dados do site da United Nations disponivel em: http://www.un.org/ (acesso em 04/07/2001).

A estrutura do sistema utilizado na Nova Zelândia apresenta particularmente um nível adicional com seis dígitos (subclasse) correspondente às indústrias específicas deste país.

\section{Classificação de Produtos}

\section{- Central Product Classification (CPC)}

A Central Product Classification Version 1.0 (CPC) é um sistema de classificação de produtos de referência que tem como gestor a United Nation Statisticsl Division (UNSD). Ele foi aprovado em 1997 pela United Nation 
Statistical Commission e substituiu a Provisional Central Product Classification (Provisional CPC), de 1989. A CPC Version 1.0 é baseada na característica física da mercadoria ou na natureza dos serviços prestados. Seu objetivo, assim como de outras classificações internacionais, é servir de instrumento de base na preparação dos dados estatísticos e, mais ainda, como classificação de referência. Espera-se que o número de classificações específicas de produtos diminua no mundo e que estas estruturas sejam compatíveis com a estrutura da CPC, buscando a harmonização das informações (United Nations, 1998).

A estrutura da CPC Version 1.0 é apresentada na tabela 8 . As seções de 0 a 4 detalham os mesmos níveis de agregação do Harmonized System (HS), um sistema de classificação de produtos utilizado em estatísticas do comércio exterior em muitos países que será apresentado em seguida. Esta correlação facilitou a implementação da CPC nos países que adotavam o HS como sistema de classificação de produtos (World Customs Organization, 1996).

O quadro 4 demonstra que a estrutura da CPC, no caso da indústria química, reflete tanto a característica do produto quanto a função química e processo de produção.

- Harmonized Commodity Description and Coding System (HS)

Na maior parte do mundo, os principais dados de estatísticas do comércio internacional são obtidos a partir dos documentos aduaneiros de importação e exportação e segundo as especificações na nomenclatura aduaneira (Funcex, 1999). Assim, tornaram-se evidentes as vantagens de uma correlação entre as classificações existentes para o sistema alfandegário, a Customs Cooperation Council Nomenclature (CCCN), e para o sistema de informação estatística, a Standard International Trade Classification (SITC).

A CCCN, que tem como órgão responsável o Customs Cooperation Council (CCC), é uma classificação aduaneira adotada por um número substancial de países com o objetivo de discriminar mercadorias para fins aduaneiros, e a SITC, que tem como órgão responsável a United Nations Statistics Division (UNSD), visa à obtenção de informações para análises estatísticas. Contudo, ambas têm por objetivo o comércio internacional, ou seja, a importação e a exportação.

Por volta de 1970, o CCC, em conjunto com a UNSD e outros órgãos estatísticos nacionais e internacionais, iniciou estudos visando a reduzir os problemas relativos à codificação e designação de mercadorias. Em 1986, o CCC aprovou o Harmonized Commodity Description and
TABELA 8

Estrutura do sistema de classificação CPC Version 1.0

\begin{tabular}{llr}
\hline Nivel & Codificação & No de níveis \\
\hline Seção & 1 dígito (0-9) & 10 \\
Divisão & 2 digitos & 71 \\
Grupos & 3 dígitos & 294 \\
Classes & 4 dígitos & 1.162 \\
Subclasses & 5 dígitos & 2.093 \\
\hline
\end{tabular}

Fonte: elaboração própria a partir de dados do site da United Nations disponível: http://www.un.org/.

\section{QUADRO 4}

\section{Exemplificação da estrutura da CPC}

3 - Outros produtos transportáveis, exceto produtos, maquinários e equipamentos metálicos (SEÇÃO)

34 - Produtos químicos básicos (DIVISÃO)

341 - Produtos químicos orgânicos básicos (GRUPO)

3411 - Hidrocarbonetos e seus derivados halogenados, sulfonados, nitrados ou nitrosados (CLASSE)

34110 - Indústria de fertilizantes (SUBCLASSE)

Fonte: United Nations, 2001 (United Nations Statistic Divisions).

Coding System (HS) ou Harmonized System (HS), uma nomenclatura comum para atender aos interesses aduaneiros e estatísticos envolvidos no comércio internacional. $\mathrm{O}$ HS é baseado em um princípio fundamental de classificação de acordo com a definição do produto, e não de acordo com seu processo de fabricação, entre outros critérios.

O sistema HS é uma classificação de produtos de referência que tem como órgão internacional responsável o CCC, conhecido também como World Customs Organization (WCO). As estatísticas de exportação e importação são classificadas e publicadas de acordo com o HS (Canada's Business and Consumer Site, 2001).

Desde 1988, o HS é, em muitos países, inclusive no Brasil, a base da pauta aduaneira, assim como das estatísticas do comércio externo. O HS compreende um total de 5.019 grupos de produtos identificados por um código de seis dígitos, dos quais os quatro dígitos iniciais correspondem à posição, enquanto o quinto e sexto dígitos correspondem às subposições, constituindo uma organização sistemática e uniforme de produtos. Há um exemplo no quadro 5, a seguir. 
Existem algumas agências alfandegárias dos EUA e do Canadá que não concordam com o código de seis algarismos do HS. Quando isto ocorre, cada agência estatística pode classificar os produtos com o código de seis dígitos designado por sua respectiva agência alfandegária* (Canada's Business and Consumer Site, 2001).

\section{- Standard International Trade Classification (SITC)}

A Standard International Trade Classification Rev 3 é um sistema de classificação de comércio exterior publicado pelas United Nations em 1985. A terceira revisão deste sistema ocorreu paralelamente à implantação do HS, e sua importância tem sido cada vez menor devido à ampla utilização do HS em todo o mundo.

A SITC Rev 3 é um sistema de classificação derivada e tem como gestor a UNSD. Seu principal objetivo é estabelecer comparabilidade entre os dados estatísticos de comércio exterior. Os grupos de produtos do SITC Rev 3, além de refletir os processos de produção utilizados na sua fabricação e as tecnologias, mostram a importância das mercadorias em termos de comércio exterior. Na tabela 9, é apresentada sua estrutura.

A seguir (quadro 6), exemplifica-se a estrutura da SITC Rev 3.

- Classification by Broad Economic Categories in Terms of the Standard International Trade Classification (BEC)

A Classification by Broad Economic Categories in Terms of the Standard International Trade Classification Rev 3 é uma classificação derivada que tem como gestor a UNSD, e seu objetivo principal é a compilação de dados estatísticos de comércio exterior, utilizando como base a SITC Rev 3, de modo a comparar com a estrutura do SNC adotado como referência pelas Nações Unidas.

Este é um tipo de classificação mais abrangente recomendado para classificar produtos de uma maneira geral, distinguindo alimentos, produtos industriais, equipamentos e bens de consumo duráveis e nãoduráveis. A BEC tinha como objetivo inicial a compilação de dados estatísticos de comércio exterior associados a estes produtos. Esta classificação passou também a ser utilizada na correlação com os dados estatísticos do Sistema de Contas Nacionais (SCN).

Em resumo, as subcategorias da BEC podem ser agregadas

\footnotetext{
* Este procedimento não é observado no Brasil, que não apresenta autonomia para mudança da classificação alfandegária.
}

\section{QUADRO 5}

\section{Exemplificação da estrutura do HS}

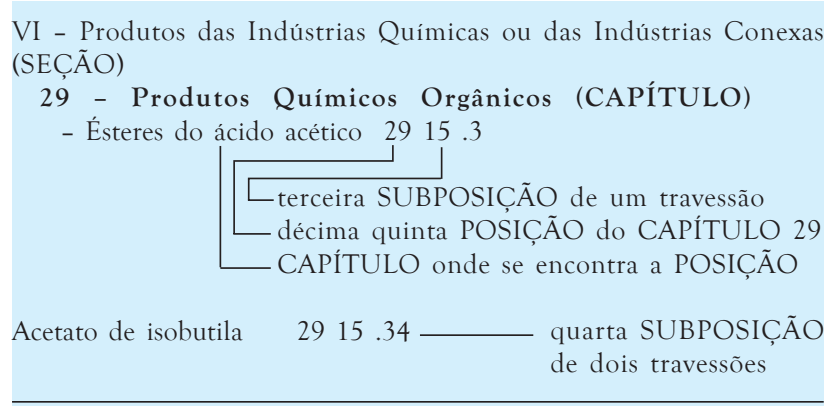

Fonte: Funcex, 2002.

\section{TABELA 9}

Estrutura do sistema de classificação SITC Rev 3

\begin{tabular}{llc}
\hline Nivel & Codificação & No de níveis \\
\hline Seção & 1 dígito (0-9) & 10 \\
Divisão & 2 dígitos & 67 \\
Grupos & 3 dígitos & 261 \\
Classes & 4 dígitos & 1033 \\
Subclasses & 5 digitos & 3121 \\
\hline
\end{tabular}

Fonte: elaboração própria a partir de dados do site da United Nations disponivel em: http://www.un.org/ (acesso em 04/07/2001).

\section{QUADRO 6 \\ Exemplificação da estrutura da SITC Rev 3}

3 - Outros produtos transportáveis, exceto maquinários e equipamentos metálicos (SEC̣ÃO)

51 - Produtos químicos orgânicos (DIVISÃO)

511 - Hidrocarbonetos e seus derivados halogenados, sulfonados, nitrados e nitrosados (GRUPO)

511.1 - Hidrocarbonetos acíclicos (CLASSE) 511.11 - Etileno (SUBCLASSE)

Obs.: este código corresponde ao HS 2901.21

Fonte: United Nations, 2001 (United Nations Statistic Divisions).

dando origem a basicamente três categorias do SCN: bens de capital, bens intermediários e bens de consumo. Isto permite que os dados estatísticos de comércio exterior sejam considerados juntamente com dados industriais e dados do SCN para análises econômicas de nível regional, nacional e internacional. Sua estrutura é simples e dividida em sete categorias representadas por um código de dois dígitos e subcategorias representadas por códigos de dois ou três dígitos (quadro 7, a seguir). 
- Statistical Classification of Products by Activity in the European Economic Community (CPA)

A CPA estabelece uma classificação de produtos de acordo com a União Européia. Constitui a base para a preparação de estatísticas da produção dos vários fatores de produção (mão-de-obra, materiais, energia etc.), de operações de investimento e financeiras de tais unidades.

A CPA é uma classificação de produtos derivada que tem como gestor o Statistical Office of the European Communities. Os elementos deste sistema encontramse relacionados com as atividades, tais como definidas pela NACE Rev 1. Todos os produtos - quer se trate de bens e serviços transportáveis ou não-transportáveis são atribuídos a uma só atividade da NACE Rev 1. A tabela 10 mostra a estrutura da CPA.

A seguir, exemplifica-se o sistema de classificação CPA (quadro 8).

\section{QUADRO 7}

Exemplificação da estrutura da BEC Rev 3

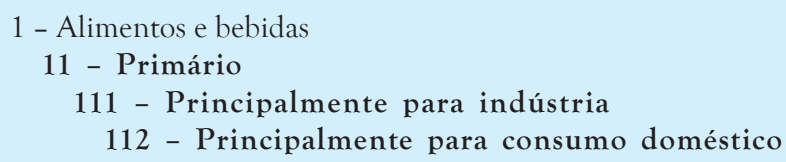

Fonte: Eurostat, 2001.

TABELA 10

Estrutura do sistema de classificação CPA

\begin{tabular}{llc}
\hline Nivel & Codificação & No de níveis \\
\hline Seção & 1 caractere alfabético (A-Q) & 17 \\
Subseção & 2 caracteres alfabéticos & 31 \\
Divisão & 2 dígitos & 60 \\
Classe & 4 dígitos & 490 \\
Categoria & 5 dígitos & 947
\end{tabular}

Fonte: elaboração própria a partir de dados do site da United Nations disponivel: http://www.un.org/ (acessado em 04/07/2001).

\section{QUADRO 8}

Exemplificação da estrutura da CPA

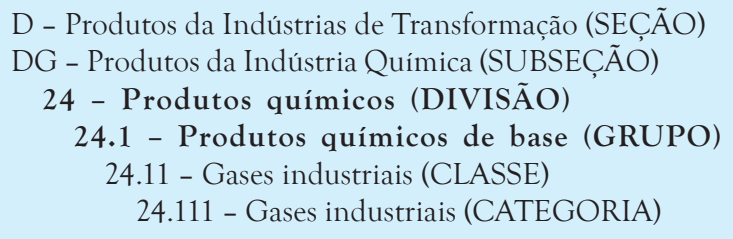

Fonte: United Nations, 2001 (United Nations Statistics Division).

\section{CONSIDERAC̣ÕES FINAIS}

A construção de estudos setoriais reflete diferentes concepções sobre o contexto socioeconômico, político e cultural no qual estes são produzidos, com impacto direto na discussão e na elaboração de políticas e tomadas de decisão por parte de diversos atores, como o governo e o empresariado. O objetivo deste artigo, parte de uma tese de doutorado intitulada "O Impacto da Indústria Química na Economia através das Contas Nacionais do IBGE", foi promover uma introdução aos pressupostos teórico-metodológicos que norteiam a utilização de classificações industriais, utilizando como base empírica a Família Internacional de Classificações Econômicas. Ao apresentar o aporte teórico envolvido na utilização de classificações internacionais de atividades e produtos em saúde, o artigo contribui para enfatizar a importância das classificações em análises estatísticas como em qualquer estudo setorial que se faça.

Artigo recebido em 17-02-2004 e aceito para publicação em 24-04-2004. 


\section{A classificação industrial e sua importância na análise setorial}

\section{REFERENCIAS}

BUREAU OF CENSUS (Washington). Disponível em: <http:// www.census.gov>. Acesso em: 04 jul. 2001.

CANADA's BUSINESS AND CONSUMER SITE. Definitions and notes on methodology: harmonized system. Disponivel em: <http:// strategis.ic.gc.>. Acesso em: 06 jul. 2001

CEFIC. European Chemical Industry Council. Disponível em: <http://www.cefic.org/>. Acesso em: 04 jul. 2001

DESROSIÈRES, A. How to make things hold together: social science, statistics and the state. In: WAGMER, P.; WITTROCK, B. (Ed.). Discourses on society: V. XV. Netherlands : Kluwer Academics, 1992. cap. 8.

EUROSTAT. Statistical Office of the European Communities. Classifications. [S. 1.], 2001. Disponível em: <http://europa.eu.int/ comm/eurostat/ramon/intro_nace_rev1/pt.html>. Acesso em: 03 jun. 2001

Office Statistique des Communautés Européennes. Nomenclature statistique des activités économiques dans la Communauté Européenne - NACE. Rev 1. Luxembourg, 1996. p. 183. (ECU, 47)

FUNCEX. Curso de comércio exterior. [S. 1.], 2001.

INSTITUTO BRASILEIRO DE GEOGRAFIA E ESTATÍSTICA (Rio de Janeiro, RJ). Diretoria de Pesquisas. Classificação de atividades e produtos, matérias-primas e serviços industriais: indústria extrativa mineral e de transformação. Rio de Janeiro, 1988. v. 1. (Textos para discussão, n. 6)

Classificações estatísticas. Rio de Janeiro, 2001. Disponível em: 〈http://www.ibge.gov.br/concla/claestat/claestat.shtm>. Acesso em: 04 jul. 2001.

Órgãos estatísticos internacionais., Rio de Janeiro, 2001. Disponível em: 〈http://www.ibge.gov.br〉. Acesso em: 04 jul. 2001 INTERNATIONAL COUNCIL OF CHEMICAL ASSOCIATIONS. Disponivel em: 〈http://www.icca-chem.org/>. Acesso em: 04 jul. 2001.

INSTITUTO NACIONAL DE ESTATÍSTICA (Rio de Janeiro, RJ). Sistemas de contas nacionais 93. Rio de Janeiro, 1998.

INSTITUTO NACIONAL DE ESTATÍSTICA, GEOGRAFIA E INFORMÁTICA DO MÉXICO. Conciliación de las estadisticas de comercio internacional de mercancias. México, 1996/1997. Disponível em: 〈http://www.inegi.gob.mx>. Acesso em: 05 jul. 2001.

INSTITUT NACIONAL DE LA STATISTIQUE E DE ETUDES ECONOMIQUES (França). Nomenclature d'activités française. [S. 1.], 2001. Disponível em: <http://www.insee.fr/fr/nomenclatures/naf/ Pages/naf.htm>. Acesso em: 15 ago. 2001.
IUPAC. Símbolos e nomenclaturas. [S. 1.], 2001. Disponível em: <http:/ /www.iupac.org/publications/books/seriestitles/nomenclature.html>. Acesso em: 07 ago. 2001.

PORCARO, R. M. Produção de informação estatística oficial na (des)ordem social da modernidade. 2000. 194 f. Tese (Doutorado em Ciência da Informação) - Escola de Comunicação da UFRJ, Rio de Janeiro, 2000.

SCHWARTZMAN, Simon. Adoption of NAICS. Disponivel em: <http:/ /www.statcan.ca/english/concepts/SNA/naics.htm>. Acesso em: 06 jul. 2002

Legitimidade, controvérsias e traduções em estatísticas públicas. In: REUNIÃO DA SOCIEDADE DE ESTUDOS SOCIAIS DA CIÊNCIA E ASSOCIACÃO EUROPÉIA PARA O ESTUDO DE CIÊNCIA E TECNOLOGIA, 1996, Bielefeld, Alemanha. [S. 1.], 1997.

Standard classifications in use at statistics Canada. Disponivel em: <http://www.statcan.ca/english/Subjects/Standard/ standard_classifications.htm>. Acesso em: 06 jul. 2002.

STATISTICS NEW ZEALAND. Methods of official statistics Disponível: <http://www.stats.govt.nz/domino/external>. Acesso em: 03 jun. 2001

UNITED NATIONS. STATISTICAL COMMISSION. New York, 1999. Disponível em: http://www.un.org. Acesso em: 03 jul. 2001

Central product classification. Version 1.0. New York, 1998. (United Nations publication. Statistical papers, Series M. n. 77). Sales n. E. 98.XVII.5.

Provisional product classification. Version 1.0. New York, 1991. (United Nations publication. Statistical papers, Series M, n. 77). Sales E.91.XVII.7.

Standard statistical classifications: basic principles. 13. ed. New York, 1999. Disponível em: 〈http://www.un.org〉. Acesso em: 03 jun. 2001.

. Statistics Divisions. New York, 2001. Disponível em: <http://www.un.org/depts/unsd/>. Acesso em: 03 jun. 2001.

VALENTE, E. Transformações estruturais e sistemas estatísticos nacionais. Rio de Janeiro : IBGE, 1996. (Textos para discussão, n. 82).

WORLD CUSTOMS ORGANIZATION (Brussels). The harmonized commodity description and coding system. Brussels, 1996. 